\title{
Capillary Desaturation Curve for Residual Nonwetting Phase in Natural Fractures
}

\author{
B. I. AlQuaimi, Delft University of Technology and Saudi Aramco, and W. R. Rossen, Delft University of Technology
}

\begin{abstract}
Summary
The displacement of a nonwetting phase by a wetting phase is characterized by the capillary number. Different forms of capillary number have been used in the literature for flow in porous media. A capillary number for a single rock fracture has been defined in the literature, using the mean aperture to characterize the trapping and mobilization in a fracture.

We propose a new capillary-number definition for fractures that incorporates geometrical characterization of the fracture, dependent on the force balance on a trapped ganglion. The new definition is validated with laboratory experiments using five distinctive model fractures. The model fractures are made of glass plates, with a wide variety of hydraulic apertures, degrees of roughness, and correlation lengths of the roughness. The fracture surfaces were characterized in detail and statistically analyzed. The aperture distribution of each model fracture was represented as a 2D network of pore bodies connected by throats. The hydraulic aperture of each model fracture was measured experimentally. Capillary desaturation curves (CDCs) were generated experimentally using water/air in forced imbibition. The transparent nature of the system permits us to determine the residual air saturation as a function of pressure gradient from the captured images.

The residual nonwetting saturation/capillary-number relationship obtained from different fractures varying in aperture and roughness can be represented approximately by a single curve in terms of the new definition of the capillary number. They do not fit a single trend using the conventional definition of the capillary number.
\end{abstract}

\section{Introduction}

Most underground reservoirs have some degree of fracturing caused by different Earth stresses. Natural fractures can be present in almost every lithology, with different characteristics in terms of their conductivity, storage capacity, and the degree of wall roughness (van Golf-Racht 1982). The reservoir is considered a naturally fractured reservoir (NFR) if fractures are predicted to have a significant effect on reservoir-fluid-flow dynamics (Nelson 2001). NFRs have been explored and exploited globally for geothermal energy, petroleum production, coalbed-methane production, and nuclear-waste sequestration (Ramspott et al. 1979; Persoff and Pruess 1995). Understanding and predicting the entire behavior of NFRs requires understanding the flow in a single fracture (Rossen and Kumar 1992). A single fracture has rough walls and variable aperture, as well as asperities where the two opposing fracture walls are in contact with each other (Olsson and Barton 2001). Therefore, it can be represented as a 2D network of locations of wide and narrow aperture (Tsang 1984; Brown and Scholz 1985; Wang and Narasimhan 1985; Brown et al. 1986; Schrauf and Evans 1986; Pyrak-Nolte et al. 1988; Morrow et al. 1990; Rossen and Kumar 1992; Odling and Roden 1997; Hughes and Blunt 2001). Thus, fractures can be considered as 2D analogs of the 3D networks of throats and bodies that compose the pore network of rock matrix (Rossen and Kumar 1992; Hughes and Blunt 2001). During two-phase flow in a fracture, there is a similar competition between viscous and capillary forces as in rock matrix, which can be represented by a capillary number. However, the capillary number in rock is not adequate to describe the mobilization of nonwetting phase in fractures, as is shown in this paper.

Rock-Matrix Capillary Number. In two-phase immiscible displacement, the competition or interplay between viscous forces and capillary forces determines the mobilization of trapped nonwetting phase. The capillary number was first described using the results of several flooding experiments by Moore and Slobod (1955) in a variety of porous media. The relationship was called the Viscap concept (Eq. 1), with "Viscap" denoting viscosity and capillary pressure:

$$
N_{c a} \equiv \frac{v \mu}{\gamma \cos \theta}
$$

where $v$ is the superficial velocity, $\mu$ is the viscosity of the displacing fluid, $\gamma$ is the interfacial tension (IFT), and $\theta$ is the contact angle. Another form of the capillary number uses the permeability of the matrix (Reed and Healy 1977),

$$
N_{c a} \equiv \frac{k|\nabla P|}{\gamma \cos \theta}
$$

where $k$ is permeability, $|\nabla P|$ is the magnitude of the pressure gradient, $\theta$ is the contact angle, and $\gamma$ is IFT. One can derive Eq. 2 from a force balance on a trapped nonwetting ganglion, assuming that both pore-throat radius and pore length each scale with the square root of permeability (Sheng 2010). This assumption is reasonable for geometrically similar porous media such as packings of beads or sand. A historical review of different forms of capillary number is presented by Taber (1981). Many of these expressions of the capillary number are equivalent (Chatzis and Morrow 1984). The major difference between different forms of capillary number is that some include the porosity and some others include relative permeability.

Fracture Capillary Number. A number of studies during the last four decades have examined fracture relative permeability and capillary pressure. The pioneering study of flow in fractures concluded that fracture relative permeability is a straight-line relationship (Romm 1966). This is a case-specific relationship that applies if capillary pressure inside the fracture is insignificant compared with

Copyright (C) 2018 Society of Petroleum Engineers

Original SPE manuscript received for review 7 May 2017. Revised manuscript received for review 3 September 2017. Paper (SPE 189448) peer approved 12 September 2017. 
gravity and viscous forces. However, when capillarity in a fracture is significant, the relative permeability deviates from linearity (Pruess and Tsang 1990; Fourar et al. 1992; Rossen and Kumar 1992; Pieters and Graves 1994; Maloney and Doggett 1997; Izadi et al. 2012).

If gravity and viscous forces are not dominant, the capillary number describes the mobilization of the nonwetting phase. Hughes and Blunt (2001) studied multiphase flow in a single fracture using a pore-network model. They generated a model of the fracture from published aperture data, and the capillary number for this model was defined as

$$
N_{c a} \equiv \frac{Q \mu_{w}}{\bar{d} b N_{y} \gamma}
$$

where $Q$ is the volumetric-flow rate, $\mu_{w}$ is the displacing-fluid viscosity (water in this case), $\bar{d}$ is the mean aperture, $b$ is the resolution (width of the pixels), $N_{y}$ is the number of pixels perpendicular to flow across the fracture, and $\gamma$ is the IFT. [We have changed the nomenclature of Hughes and Blunt (2001) for consistency with our derivation later in this text.] This definition is obtained from Eq. 1 by replacing the superficial (Darcy) velocity with the volumetric-flow rate $Q$ divided by the cross-sectional area $\left(\bar{d} b N_{y}\right)$. The derivation of the capillary number for rock from a force balance on a trapped ganglion assumes that permeability scales with the product of porethroat radius and pore-body length. This assumption is questionable for fractures, where fracture permeability could be the same for a slit with smooth walls and no trapping and a fracture with large variations in aperture and significant trapping.

We present a derivation of the capillary number for a fracture dependent on force balance on a trapped nonwetting ganglion. The variation of aperture $d$ is the geometric parameter that is responsible for trapping the nonwetting phase in the fracture. Capillary pressure across a curved interface where the aperture is $d$ is

$$
P_{c}=\frac{2 \gamma \cos \theta}{d}
$$

Eq. 4 assumes that the length scale along which aperture varies in the fracture plane is much greater than the aperture itself; thus, interfaces are nearly cylindrical rather than spherical. We provide justification below. The principal radii of curvature of the interface between wetting and nonwetting phases are thus $r_{1}=d \cos \theta / 2$ and $r_{2} \approx \infty$ (Pruess and Tsang 1990). Consider a fracture with some degree of roughness where a trapped ganglion is on the verge of forward displacement, as shown in Fig. 1.

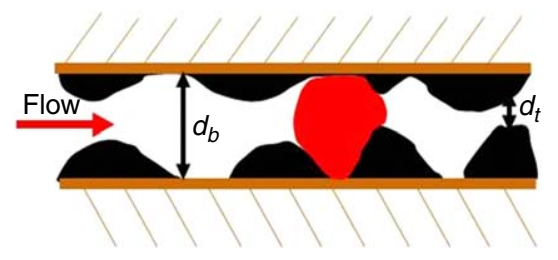

Fig. 1-Schematic of rough fracture with a trapped ganglion. The aperture of the fracture is greatly exaggerated in this figure compared with distance along the fracture plane.

Fig. 1 shows the flow direction and the characteristic pore-throat and pore-body apertures. The aperture is exaggerated in Fig. 1. As noted, the curvature across the fracture is much greater than that along the fracture; therefore, the maximum capillary pressure during passage through the throat can be written as

$$
P_{c}=\frac{2 \gamma \cos \theta}{d_{t}}
$$

where $d_{t}$ is the minimum aperture (i.e., aperture at the throat). The capillary pressure difference across the interfaces of a ganglion, with its leading edge penetrating a throat and its trailing edge in a pore body, is given by

$$
\Delta P_{c}=\left(\frac{2 \gamma}{d_{t}}-\frac{2 \gamma}{d_{b}}\right) \cos \theta
$$

where $d_{b}$ is the aperture at the body. The pressure difference across the ganglion, with length of $L_{g}$, must be greater than this pressure difference if the ganglion is to be mobilized:

$$
\nabla P L_{g}>\left(\frac{2 \gamma}{d_{t}}-\frac{2 \gamma}{d_{b}}\right) \cos \theta=\frac{2 \gamma}{d_{t}}\left[1-\left(\frac{d_{t}}{d_{b}}\right)\right] \cos \theta .
$$

One can regroup the terms in Eq. 7 to restate the criterion for mobilization in terms of a dimensionless capillary number:

$$
\frac{\nabla P L_{g} d_{t}}{2 \gamma\left[1-\left(\frac{d_{t}}{d_{b}}\right)\right] \cos \theta} \equiv N_{c a}>1
$$

The permeability of a fracture, approximated as a smooth rectangular slit, can also be written as a function of the hydraulic aperture (van Golf-Racht 1982; Tsang 1992; Zimmerman and Bodvarsson 1996):

$$
Q=\frac{1}{12} \frac{|\nabla P| w d_{H}^{3}}{\mu} ; k_{f} \equiv \frac{d_{H}^{2}}{12},
$$

where $Q$ is the volumetric-flow rate, $|\nabla P|$ pressure gradient, $w$ is the width perpendicular to flow, $\mu$ is the viscosity, and $d_{H}$ is the hydraulic aperture. 
Eq. 9 is in effect a definition of the hydraulic aperture $d_{H}$. Introducing permeability $k_{f}$ into Eq. 8 , and noting its relation to $d_{H}$, yields

$$
\begin{gathered}
\frac{L_{g} d_{t}}{1-\left(\frac{d_{t}}{d_{b}}\right) \cos \theta} \equiv\left(d_{H}\right)^{2}\left(\frac{d_{t}}{d_{H}}\right)\left(\frac{L_{g}}{d_{t}}\right)\left(\frac{d_{t}}{d_{H}}\right) \frac{1}{1-\left(\frac{d_{t}}{d_{b}}\right) \cos \theta}, \\
N_{c a} \equiv\left(\frac{\nabla P k_{f}}{\gamma \cos \theta}\right)\left[\left(\frac{12}{2}\right)\left(\frac{d_{t}}{d_{H}}\right)^{2}\left(\frac{L_{g}}{d_{t}}\right) \frac{1}{\left.1-\left(\frac{d_{t}}{d_{b}}\right)\right] \ldots . .}\right.
\end{gathered}
$$

The first part of this definition of the capillary number is identical to Eq. 2 (i.e., the definition used traditionally for porous media). The second part accounts for the effect of fracture roughness: the narrowness of the "throats," the distance between throats, and the contrast in aperture between pore throats and bodies. The term in brackets is a geometric factor; Yeganeh et al. (2016) identified a similar factor in the capillary number for rock matrix. To be useful, the terms in this definition must be derivable from a consideration of the fracture itself, without, for example, needing to conduct a two-phase-flow experiment (Tsang 1984; Brown and Scholz 1985; Wang and Narasimhan 1985; Brown et al. 1986; Schrauf and Evans 1986; Pyrak-Nolte et al. 1988; Morrow et al. 1990; Johns et al. 1993; Hakami and Larsson 1996; Odling and Roden 1997; Oron and Berkowitz 1998; Hughes and Blunt 2001; Karpyn et al. 2007; Lang et al. 2015, 2016). We describe how to drive these parameters in the next section.

\section{Design of Model Fracture}

Five different physical-model fractures were designed for capillary desaturation experiments to test the new expression for the capillary number. In the literature, different experimental approaches are presented, including splitting the core into two halves, which are then reassembled with a spacer in between (Haugen et al. 2012; Skoreyko et al. 2012). However, one cannot observe directly the flow inside an opaque rock sample. Microfluidic devices are used to represent flow in fractures (Buchgraber et al. 2012; Gauteplass et al. 2015). Microfluidic devices have distinct, usually rectangular, channels, which do not represent an aperture that varies continuously over space. A third option is to design a fracture apparatus made of glass plates. Model fractures using glass plates have been used to study flow in fractures (Fourar et al. 1992; Pieters and Graves 1994; Chen et al. 2004a, b; Yan et al. 2006; Qian et al. 2011). The transparent nature of the glass provides the ability to observe the flow and to determine the saturation during the experiment. The variety of commercially available roughened glass makes it possible to test a wide range of types and scales of roughness. Therefore, we designed five different model fractures using glass plates fabricated in collaboration with Trace Elemental Instruments (Delft, The Netherlands).

The lengths of the fractures are $30 \mathrm{~cm}$ with a width of $10 \mathrm{~cm}$ perpendicular to flow. Each fracture consists of a roughened plate that represents fracture-wall roughness and a top plate that is smooth, to allow direct observation of the flow and measurement of saturation. Here, as in other studies (Pieters and Graves 1994; Chen et al. 2004a, b), area fraction is used as an approximation to fracture saturation. Five different roughened-glass samples were selected for this study, with a wide variety of degrees of roughness. Each roughened plate includes four ports, two to measure the pressure difference and two as flow inlet and outlet. The gap between the top plate and the rough surface represents the fracture aperture. The two glass plates are glued together using Araldite 2014 (Polyestershoppen B.V., Moordrecht, The Netherlands) which is a two-component epoxy adhesive that has a tensile strength of $26 \mathrm{MPa}$ at $23^{\circ} \mathrm{C}$. The glue was found not to have any adverse effect on IFT. The plates are mounted using two wooden bars to hold the plates firmly and prevent the glass from bowing outward during the flow.

Geometric Characterization of Model Fractures. The five roughened-glass samples were profiled to quantify the spatial and vertical variation in the height of the surface of each sample. The Chromatic Profiler (Precitec Optronik GmbH, Philips Innovation Services, Eindhoven, The Netherlands) has a height resolution of $0.5 \mu \mathrm{m}$ and scanning window of $50.0 \times 50.0 \mu \mathrm{m}$. The device uses a lens system with chromatic aberration, and reflected color is a measure for height. A randomly selected patch of area of $4.0 \times 4.0 \mathrm{~cm}$ was used for the measurement. Light scattering causes the optical profilers to miss the determining height at some locations. It accurately captures height at positions with relatively shallow small slopes, but not at positions with very steep slope. Fortunately, the regions captured include the maxima, minima, and saddle points in height, which are the most significant locations for our purposes. The instrument restores the missing data using spline interpolation. We limit the restoring algorithm to five iterations to avoid creating spurious maxima and minima in our measurements. We scanned two separate $4.0 \times 4.0$-cm patches in the same sample to confirm that we had obtained a representative measurement for the whole surface. Fig. 2 shows the results of the two areas measured in Sample 1.

In addition, the descriptive statistics of each patch were calculated along with the height values obtained from the Chromatic Profiler. The shapes of the height-data histograms of the two areas are similar (Fig. 3), which indicates that although the surface features are random, they are statistically similar.

Some descriptive statistics of the two measurements are shown in Table 1. $S_{a}$ and $S_{q}$ are the arithmetic and root-mean-square deviation from average height, respectively. They are within $5 \%$ of each other at two randomly selected patches. $S_{a}$ and $S_{q}$ are calculated using Eqs. 12 and 13, respectively, where $A$ is the area of a pixel and $z$ is the height.

$$
\begin{aligned}
S_{a} & =\frac{1}{A} \iint_{A}|z(x, y)| \mathrm{d} x \mathrm{~d} y, . \\
S_{q} & =\sqrt{\frac{1}{A} \iint_{A} z^{2}(x, y)} \mathrm{d} x \mathrm{~d} y .
\end{aligned}
$$

From this analysis, we conclude that the $4.0 \times 4.0-\mathrm{cm}$ patch is adequate to represent the roughness of the whole surface. The remaining four samples were scanned using NPFLEX White Light Interferometer Optical Profiling (Philips Innovation Services, Eindhoven, The Netherlands), which has an accuracy of 0.1 to $3 \mathrm{~nm}$ depending on the measuring technique, and the data processing was performed with MountainsMap 6.2 (Digital Surf, Besancon, France). The white-light interferometer was used because the Chromatic Profiler provided unsatisfactory results in terms of both coverage and the expected height data. Two samples out of four were coated by depositing a layer of silver that was $150 \mathrm{~nm}$ thick to enhance the reflection. Sample 2 is regular pattern, and hence the measurement was performed 
on $1.0 \times 1.0 \mathrm{~cm}$ of the glass and a lateral resolution of $3.6 \mu \mathrm{m}$. Fig. 4 shows the 3D surface topography of Sample 2 . Figs. 5, 6, and 7 show the 3D surface topography for Samples 3, 4, and 5, respectively. The surface height differs substantially among the samples. The zero value is an arbitrary height that is defined as the average height after leveling. Table 2 summarizes all sample-height statistics and roughness values.
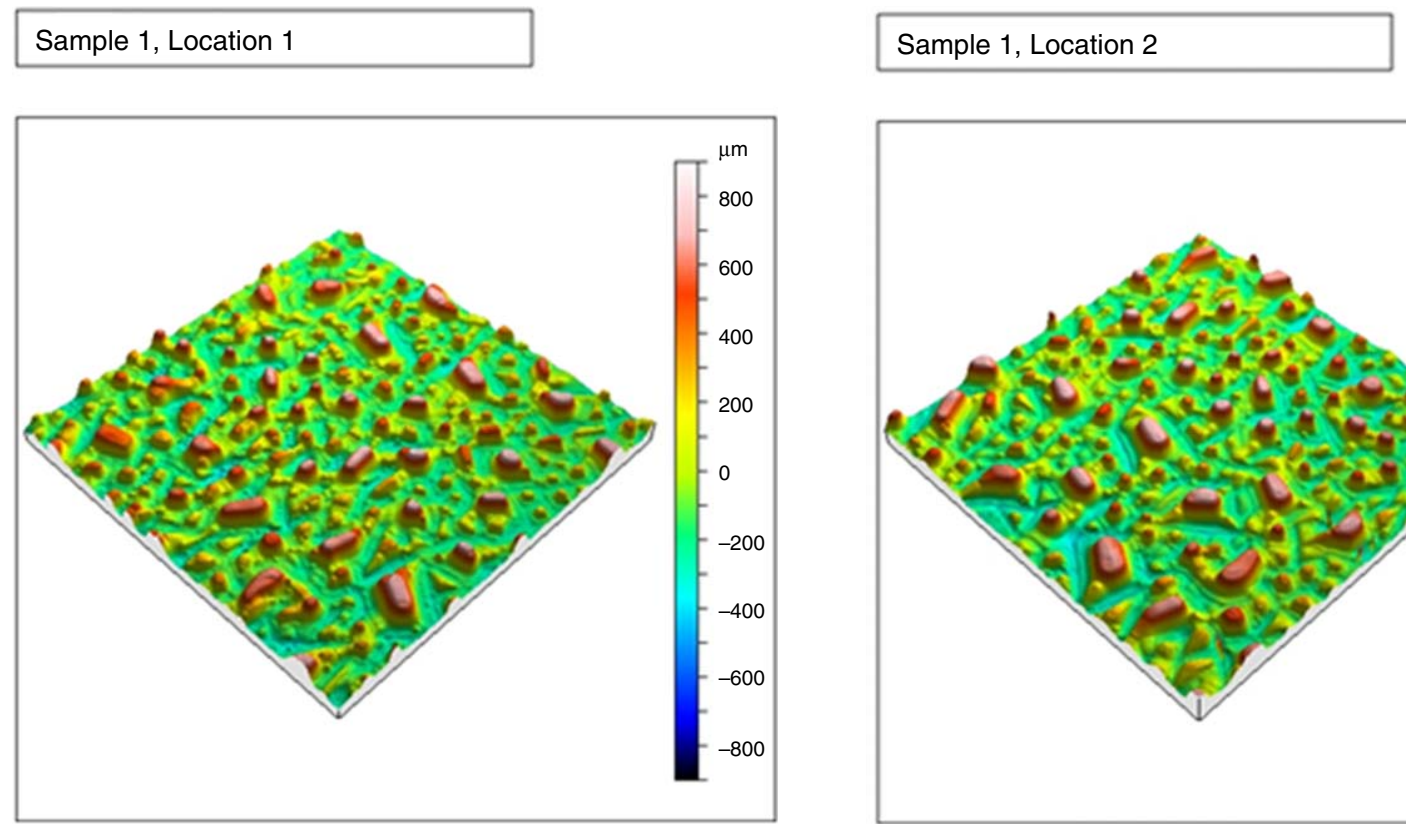

Fig. 2-Chromatic Profiler height data: (left) Patch 1; (right) Patch 2.
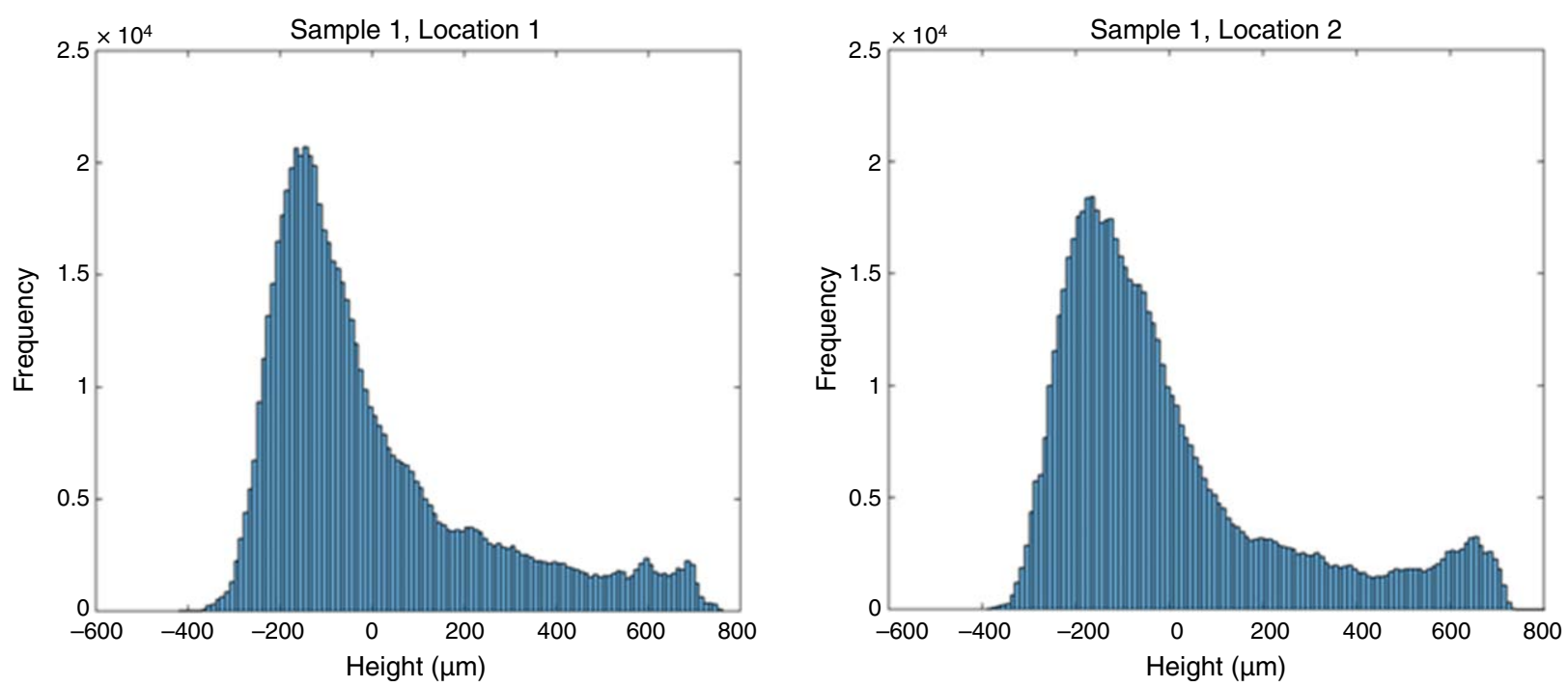

Fig. 3-Histogram of the height data of Sample 1: (left) Patch 1; (right) Patch 2.

\begin{tabular}{lcc} 
& Location 1 & Location 2 \\
\hline Mode & -189 & -188 \\
Median & -77 & -77 \\
$S_{a}$ Eq. 12) & 189 & 180 \\
$S_{q}$ (Eq. 13) & 247 & 233 \\
\hline
\end{tabular}

Table 1-Sample 1 height statistics and roughness values.

Fracture 2D Flow Network. Mobilizing or trapping a ganglion of dispersed phase depends on both the aperture at the restriction in flow and the length of the ganglion. A fracture can be considered as a 2D network of pore bodies (maxima in aperture) connected by throats (saddle points between pore bodies). Several methods are available in the literature to extract a realistic pore network of matrixrock samples, as described by Rabbani et al. (2014). The method we use is derived from a simple concept using flood-fill and image 
slicing. A MatLab ${ }^{\circledR}$ (MathWorks, Inc., Eindhoven, The Netherlands) code was developed to highlight all areas with height less than some threshold and then produce images at every $5-\mu \mathrm{m}$ increment in height for the very rough samples and at $10-\mu \mathrm{m}$ increments for the less-rough ones. An isolated, deep region represents a pore body. When two regions join upon increasing height, the connection between them is a pore throat. The sequence of images is loaded into ImageJ, a public-domain, Java-based image-processing program (developed at the US National Institute of Health), to identify the pore throats and draw the pore-body boundaries. The pore-body depth is the deepest point surrounded by at least two throats. AlQuaimi and Rossen (2017) show histograms of pore-throat and pore-body heights. The constrictions to flow are not local maxima in height, but saddle points between them.

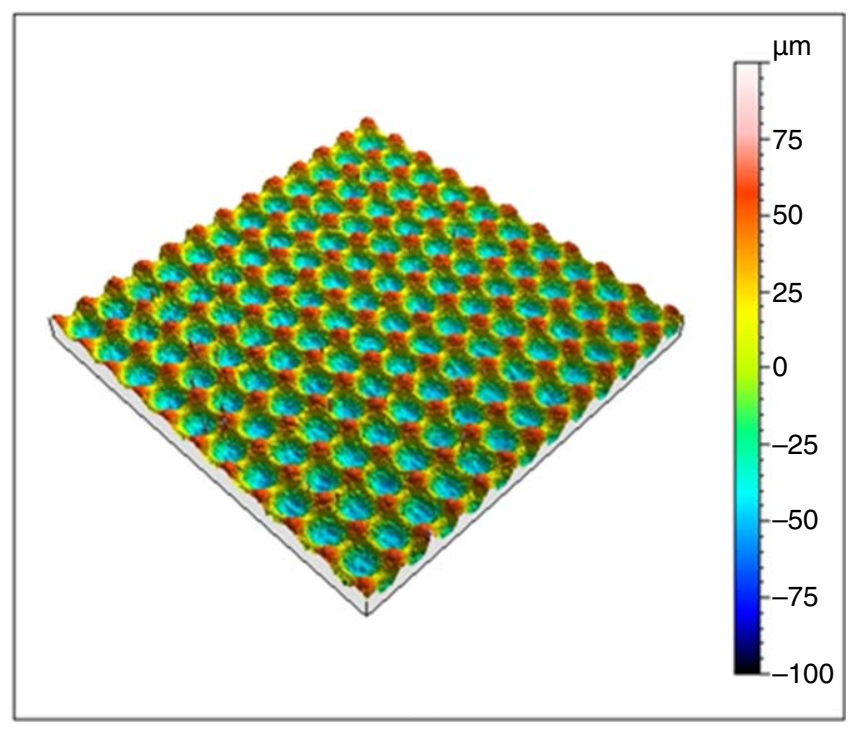

Fig. 4-Sample 2, 3D surface topography.

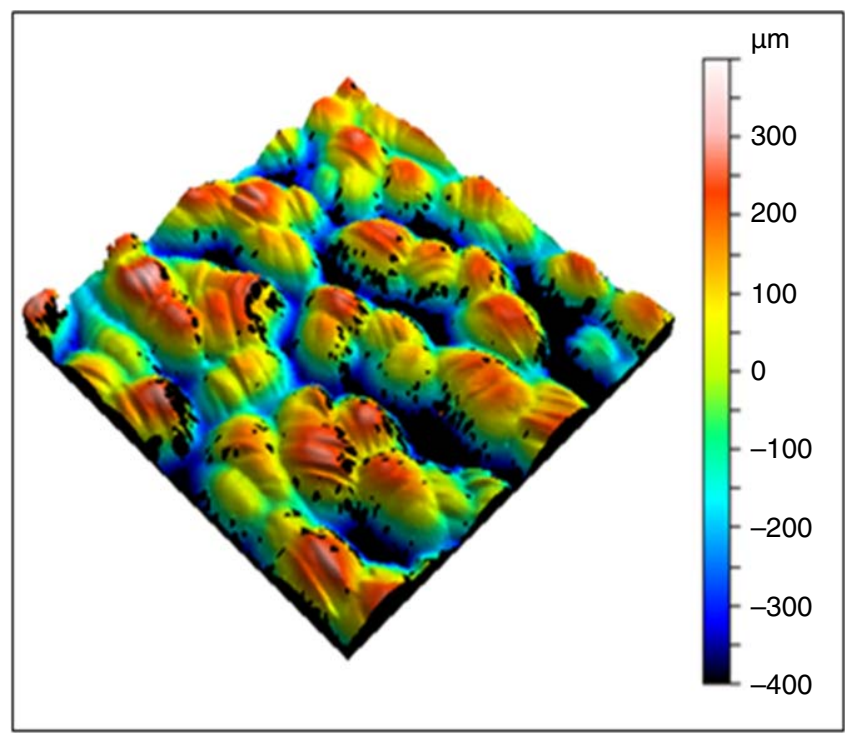

Fig. 5-Sample 3, 3D surface topography.

The pore-throat and pore-body identifications were superimposed on the 3D topographic images of all the samples to generate the 2D networks. AlQuaimi and Rossen (2017) show pore-throat and pore-body locations on the fracture topography. Appendix A shows the 2D networks of pores constructed in this way. The lines are drawn around the pore bodies and connecting the pore throats of each pore. In some cases, we exclude shallow saddle points within a pore body and count them as part of the larger pore body. The 2D network was also used to estimate the characteristic length of a pore body, which is necessary for our definition of the capillary number (Eq. 11). The length is measured in two directions for all the pore bodies and then an average value is used in the analysis. Semivariogram analysis was also conducted to quantify the special variation of the roughness. The correlation length for aperture is an alternative measure of ganglion length, as shown here. AlQuaimi and Rossen (2017) show the semivariogram analysis of our model fractures. The distance between two consecutive peaks or troughs in the semivariogram is the correlation distance.

\section{Experimental Design}

The $30.0 \times 10.0-\mathrm{cm}$-long model fracture consists of two glass plates, a clear-glass plate on the top and a rough-glass plate on the bottom, glued together at the edges with adhesive. Two wooden holders are screwed on the fracture to prevent the glass from bowing 
outward, which could cause the aperture to vary. The bottom glass plate has injection and production ports, and two pressure ports with a distance of $16 \mathrm{~cm}$ in between. A pressure-difference sensor is connected to the model fracture to provide pressure readings across an area of $16.0 \times 10.0 \mathrm{~cm}$. The sensors are silicon on-chip, signal conditioned, and temperature compensated. These MPXV5010DP and MPXV5010DP transducers (Freescale Semiconductor, Muenchen, Germany), have a range of 0 to $10 \mathrm{kPa}$ and 0 to $50 \mathrm{kPa}$, respectively, with a maximum error of $5.0 \%$ from 0 to $85^{\circ} \mathrm{C}$ temperature. The sensors are connected to a data-acquisition unit and a computer, where pressure is recorded once per second.

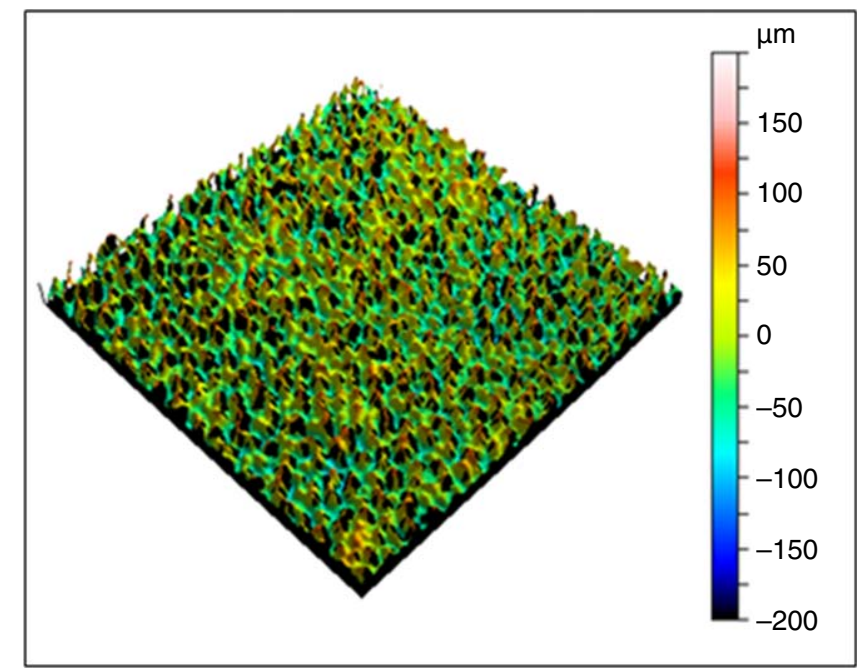

Fig. 6-Sample 4, 3D surface topography.

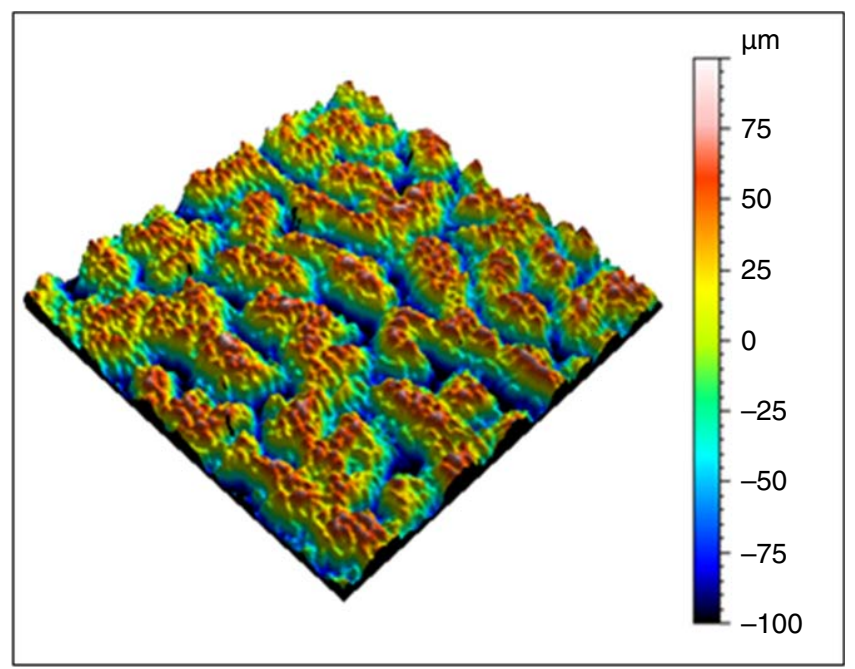

Fig. 7-Sample 5, 3D surface topography.

\begin{tabular}{lccccc} 
& Sample 1 & Sample 2 & Sample 3 & Sample 4 & Sample 5 \\
\hline Mode & -189 & -42 & 94.2 & 22.7 & -31.7 \\
Median & -77 & -0.5 & 21.7 & 7.5 & -6.9 \\
$S_{a}$ (Eq. 12) & 189 & 29.1 & 131 & 36 & 35 \\
$S_{q}$ (Eq. 13) & 247 & 33.7 & 157 & 44 & 42 \\
\hline
\end{tabular}

Table 2-Summary of all samples' height statistics and roughness values.

Water is injected through a standard infusion PHD Pump Model-703005 (Harvard Apparatus, Holliston, Massachusetts, USA). Flow rates are stated to be accurate within $0.35 \%$, with reproducibility within $0.05 \%$. This pump is equipped with microstepping techniques to further reduce flow pulsation. The pumping rate ranges from $0.0001 \mu \mathrm{L} / \mathrm{h}$ to $220 \mathrm{~cm}^{3} / \mathrm{min}$.

A high-definition camera is placed on top of the fracture to capture images at different stages during the experiment.

A compact backlight (Model CVI STAR-BL-110/110-WH-24V, Stemmer Imaging, Zutphen, The Netherlands) provides constant and even illumination. Uniform light is needed to produce noise-free images, which are used to obtain the saturation at different pressure gradients. The whole setup is placed in a $60 \times 60 \times 150-\mathrm{cm}$ box to isolate the system from external lights, as shown in Fig. 8 . 


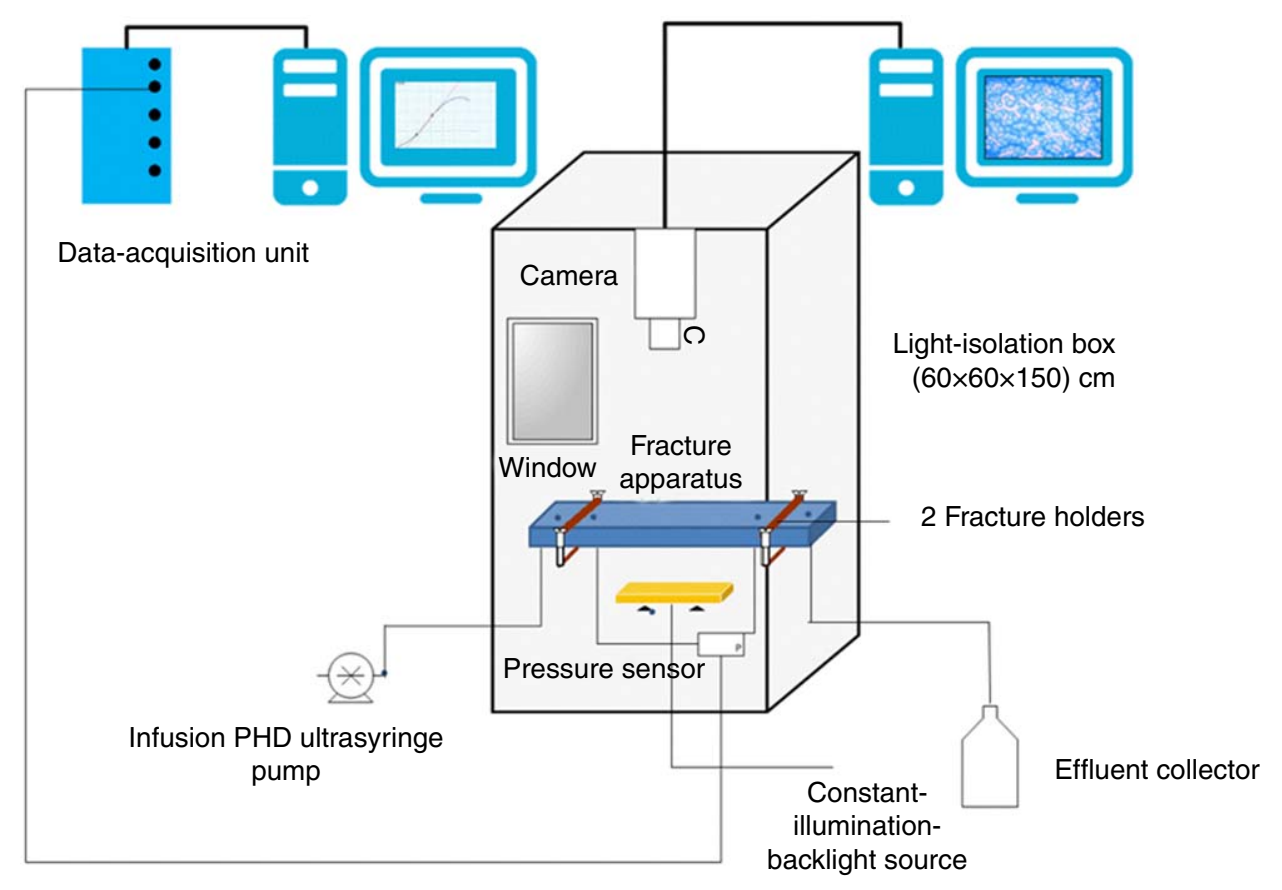

Fig. 8-Experimental-setup layout.

The setup is used first for measuring the hydraulic aperture, dependent on single-phase water flow, followed by capillary desaturation experiments.

Determination of Hydraulic Aperture. To determine aperture from the height data, one must estimate the gap between the flat upper glass plate and the peaks in the roughened plate. Fluid flow through a single fracture has been described approximately by Eq. 9 (Witherspoon et al. 1980; Hakami and Larsson 1996), which assumes that the fracture comprises two smooth, parallel plates. The hydraulic-aperture values were measured experimentally by injecting water and measuring the pressure gradient.

The hydraulic-aperture values and the distribution of the height values were used with effective medium approximation (EMA), which provides an approximate solution of the conductivity of heterogeneous network by converting it into a hypothetical homogeneous one with conductivity $g_{m}$ (Kirkpatrick 1973 ; Koplik 1981, 1982; Rossen and Kumar 1992), to estimate the gap distance $\left(d_{z}\right)$ between the highest point of the rough plate and the flat top plate. If $d_{z}$ is zero, then the two plates are estimated to be in contact at the peaks of the roughened plate. Therefore, no adjustment is needed for $d_{t}$ and $d_{b}$ in Eq. 14. $d_{z}$ was estimated by comparing the hydraulic aperture from the experiments to that estimated using EMA and aperture distribution:

$$
\int_{0}^{\infty} n(d) \frac{g_{m}-g(d)}{g(d)+\left(\frac{Z}{2}-1\right) g_{m}} \mathrm{~d} d=0,
$$

where $n(d)$ is the area fraction from the histogram of each surface, $g(d)$ is the conductivity $\left(d^{3}\right)$ of aperture $d$ at a particular location, $Z$ is the coordination number of the network, and $g_{m}$ is the effective conductivity of the medium (i.e., $d_{H}^{3}$ ). The value of $Z$ was selected to be 4. This value was used previously to study single- and two-phase flow in a fracture (Rossen and Kumar 1992). The distance $d_{z}$ was adjusted until Eq. 14 was satisfied with $g_{m}=d_{H}^{3}$.

The experiments start with a small water-flow rate, and the rate is increased to obtain several pressure measurements. The flow experiments for our model fractures showed a linear relationship between $Q$ and $|\nabla P|$, which indicates that the inertial forces were negligible and there was no change in aperture during flow. This linear relationship is used in Eq. 9 to obtain the hydraulic aperture. AlQuaimi and Rossen (2017) show the relation between flow rate and pressure gradient. The sensor was not able to record any pressure gradient in Sample 1 using water flow. Therefore, a 70 wt\% glycerol (49770 Sigma-Aldrich; Sigma-Aldrich Chemie BV, Zwijndrecht, The Netherlands) solution was used, after measuring its viscosity using an Anton Paar Rheometer (Anton Paar GmbH, Oslo, Norway). The viscosity of the solution was $0.0251 \mathrm{~Pa} \cdot \mathrm{s}$.

Table 3 provides the values of the hydraulic aperture. The hydraulic-aperture values and the distribution of the height values were used using the EMA (Eq. 14) to estimate $d_{z}$, as also given in Table 3.

\begin{tabular}{lccccccc} 
& $d_{H}(\mu \mathrm{m})$ & $d_{z}(\mu \mathrm{m})$ & $d_{b}(\mu \mathrm{m})$ & $d_{t}(\mu \mathrm{m})$ & $L_{P}(\mu \mathrm{m})$ & $L_{\text {cor }}(\mu \mathrm{m})$ & Geometric Factor \\
\hline Sample 1 & 660 & 0 & 1118 & 808 & 2661 & 2754 & 106.8 \\
Sample 2 & 79 & 8 & 138 & 68 & 819 & 795 & 108.3 \\
Sample 3 & 324 & 54 & 847 & 437 & 5156 & 4800 & 258.5 \\
Sample 4 & 116 & 45 & 255 & 145 & 4415 & 5100 & 661.7 \\
Sample 5 & 102 & 0 & 198 & 118 & 2421 & 2240 & 407.7 \\
\hline
\end{tabular}

Table 3-A summary of the geometric parameters and the geometric factor of the model fractures. 
We take the characteristic pore-throat aperture $d_{t}$ in Eq. 11 to be the aperture at the percolation threshold of this network. This is the height at which the fluid forms a continuous path across the patch in a specific direction. The flood-fill images were used to determine the percolation threshold. AlQuaimi and Rossen (2017) illustrate the percolation threshold for each sample. We take the average porebody aperture for $d_{b}$, and the average length of the pores $\left(L_{p}\right)$ to be $L_{g}$. A simpler approach, and equally accurate, is to take the correlation length $\left(L_{\mathrm{cor}}\right)$ of the aperture distribution for $L_{g}$. Individual ganglia may differ in length from $L_{p}$ or $L_{\mathrm{cor}}$, but on average they are expected to scale with either measure, as in 3D porous media (Larson et al. 1981; Chatzis et al. 1983; Mayer and Miller 1992). Table 3 summarizes the values of the characteristic pore-throat aperture $\left(d_{t}\right)$, characteristic pore body-aperture $\left(d_{b}\right)$, and the pore-body length estimated using pore-network analysis $\left(L_{p}\right)$ and the semivariogram $\left(L_{\text {cor }}\right)$. The difference in length of the pore body between the two different procedures is modest. The pore-body length that is used in the calculation in the capillary number (Eq. 11) is the one derived from the pore-network analysis. Table 3 also shows that the ratio $\left(d_{t} / L_{p}\right)$ ranges from approximately 3 (Sample 1$)$ to approximately 30 (Sample 4). The width of a throat in the fracture plane scales with (but is somewhat smaller than) $L_{p}$; this indicates that for all the fractures except for Sample 1, our assumption in Eq. 6 that interface curvature across the fracture aperture is much greater than that within the fracture plane is very good.

Numerous studies characterizing aperture variation and fracture-wall roughness could be used to generate such a representation (Tsang 1984; Brown and Scholz 1985; Wang and Narasimhan 1985; Brown et al. 1986; Schrauf and Evans 1986; Pyrak-Nolte et al. 1988; Morrow et al. 1990; Johns et al. 1993; Hakami and Larsson 1996; Odling and Roden 1997; Oron and Berkowitz 1998; Hughes and Blunt 2001; Karpyn et al. 2007; Lang et al. 2015, 2016).

\section{Capillary Desaturation Experiment}

Water/air forced-imbibition experiments were conducted with dyed water to enhance the contrast between the air and water. The dye was methylene blue (Fluka Chemie AG, Buchs, Switzerland), with a concentration of $0.001 \mathrm{wt} \%$. The surface tension of the dyed water was $70.0 \mathrm{mN} / \mathrm{m}$, as measured by KSV Sigma Tensiometer (Dyne Testing Limited, Staffordshire, UK). We assume cos $\theta=1$ (perfectly wetting by water) in the calculation of the new capillary number (Eq. 11), but at any case it is constant throughout the experiments. In these experiments, the procedure is as follows. The model-fracture parts are thoroughly cleaned using ethanol before fabrication. The injected water is demineralized to avoid mineral precipitation. The syringe and tubes are changed for each model-fracture experiment. Water is injected at $0.5 \mathrm{~cm}^{3} / \mathrm{min}$, in horizontal flow, until no further change in saturation is observed. The rate is increased and an image is taken when two conditions are satisfied: The first condition is that no further change in saturation is observed, and the second is that the pressure is stable for at least 15 minutes. Successive images are taken with incremental increases in injection rate until low residual saturation is achieved. The images are loaded into the image-processing software ImageJ to determine the saturation at each pressure gradient.

The hydraulic aperture of Samples 1 and 3 are the largest, as shown in Table 3; therefore, no measurable pressure gradient was recorded with water in Sample 1, and insignificant pressure gradient in Sample 3. A glycerol mixture was therefore used instead. Methylene blue-dyed water and glycerol appeared to be unstable to phase separation. Thus, a glycerol-based food coloring was used for these samples instead of methylene blue. The new mixture was stable and this dye has only a minor effect on the surface tension, as shown in Table 4. Precipitation was checked by measuring the surface tension and observing sample appearance after the mixing and 24 hours afterward.

\begin{tabular}{lcc} 
Mixture & $\gamma$ (dynes/cm) & Comment \\
\hline Glycerol (water 30\% + methylene blue) & 64 through 52 & Not stable over 0.5 hours \\
Glycerol (water 30\%) & 66.3 & Recently mixed \\
Glycerol (water 30\%) & 66.2 & Idle for 24 hours \\
Glycerol (water 30\%+ glycerol dye) & 65.9 & Recently mixed \\
Glycerol (water 30\% + glycerol dye) & 65.6 & Idle for 24 hours \\
\hline
\end{tabular}

Table 4-Surface-tension measurement of glycerol mixture.

Results. AlQuaimi and Rossen (2017) show the unprocessed and processed images of all the desaturation tests. An example of the unprocessed and the processed images is shown in Appendix B. We developed two procedures for the analysis of the images: image thresholding to detect the boundary of the ganglion and the built-in finding-edges option in ImageJ. The difference between these two procedures is used as the estimated error in the analysis of the saturation (Fig. 9; $y$-axis). The estimated error in $N_{c a}$ (Fig. 9; $x$-axis) reflects fluctuations in the measurements of the pressure sensor. Fig. 9 shows the relation between air saturation normalized to initial saturation and pressure gradient. In a few cases, at relatively low pressure, gradient gas trapped upstream was displaced into the region we monitored and became trapped there; thus, in a few cases normalized saturation increases before it declines with increasing pressure gradient.

The pressure gradient $|\nabla P|$ required to mobilize ganglia and the rate of change of saturation differ among samples. The aperture size and the length scale along which aperture varies explain the difference in pressure gradient required to mobilize ganglia in the five model fractures. Fig. 10 shows the CDC of the five samples plotted against the conventional capillary number (Eq. 2) plotted in a semi$\log$ scale (Larson et al. 1981; Lake et al. 1986; Sheng 2010). The scatter is less than in Fig. 9, but still the trend varies by at least an order of magnitude in $N_{c a}$. Using the experimental data along with the geometric factor we determined for the individual model fractures (Eq. 11), the relationship can be represented approximately by a single curve (Fig. 10). The trends for four of the samples overlie each other, and Sample 2 differs from the others by much less than an order of magnitude. Sample 2 is the narrowest of our model fractures, and the only one with a regular, repeating pore structure. In other respects, it falls within the range of properties of the other samples. Beyond this observation, we do not have an explanation for the difference in our results. Nevertheless, the difference is still less than scatter in the plot using conventional capillary number. The new capillary number falls in a higher range because of the geometric factor. The new definition derived from a force balance on a ganglion trapped in a fracture better represents the mobilization of the nonwetting discontinuous phase in the fracture by using the geometric parameters for the fracture. As noted, means exist to measure the parameters required for this geometric factor. Alternatively, it may be possible to develop heuristics to relate fractures of different types (shear or open fractures) or in different geological formations to these parameters, similar to how $N_{c a}$ correlations in rock are adjusted for different formations (Larson et al. 1981; Lake et al. 1986). 


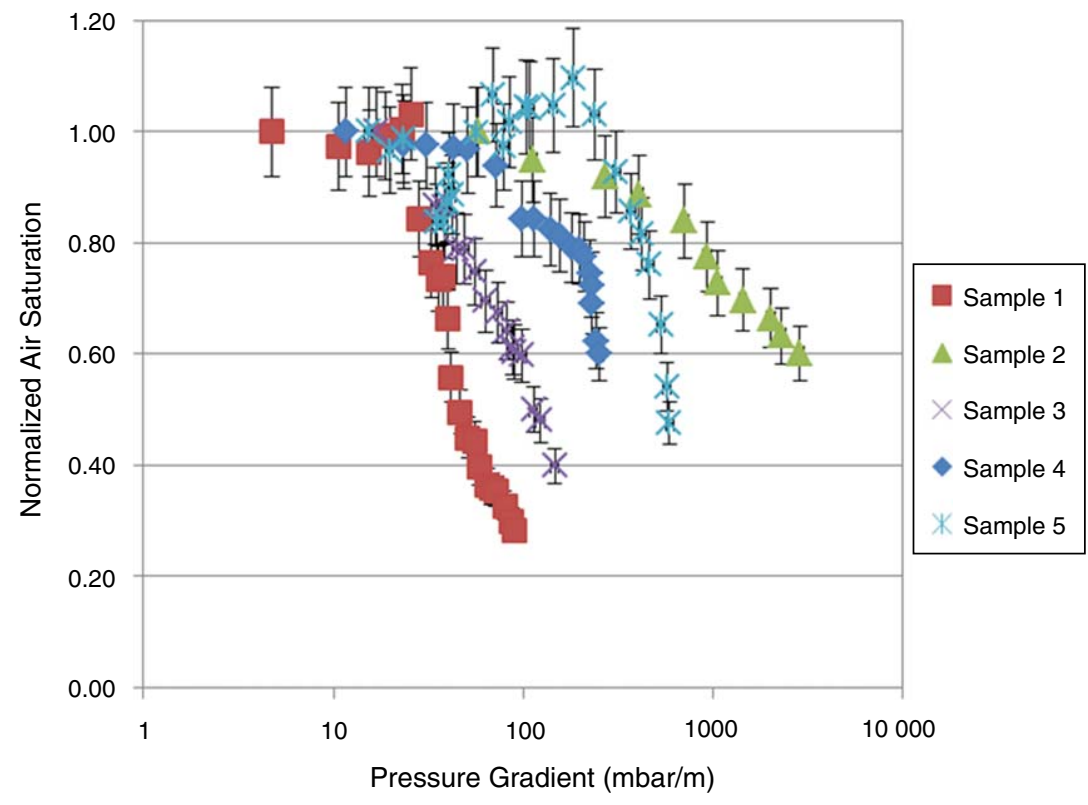

Fig. 9-Normalized air saturation in experiments vs. pressure gradient for the five different model fractures. The estimated error in the $y$-axis represents the uncertainty in the analysis of images of trapped air, whereas the estimated error in the $x$-axis represents the maximum error of the pressure sensor.

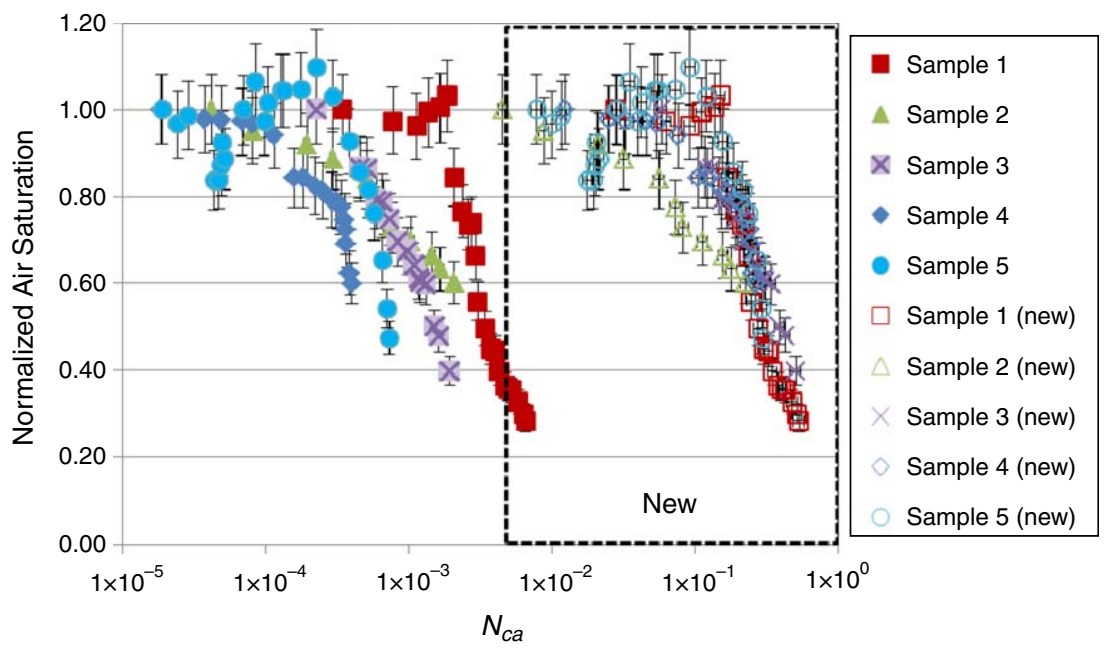

Fig. 10-Comparison between conventional (Eq. 2) and new capillary numbers (Eq. 11). The trend varies considerably between samples. The plot shows that the trend cannot be captured by the conventional capillary number. The relationship can be represented by approximately a single curve if the defined fracture geometric parameters are considered.

\section{Conclusions}

1. A new definition of capillary number for fractures was defined from a force balance on a trapped ganglion in a fracture.

2. Three geometric parameters were identified to describe features governing mobilization. These parameters can be determined from analysis of a map of fracture aperture along the fracture, with no two-phase-flow data.

3. A 2D pore-network representation of each fracture was generated from the map of aperture using flood-fill and image-slicing techniques.

4. Experimental desaturation experiments were conducted to quantify the relationship between trapped ganglions and the pressure gradient.

5. The new definition of the capillary number was tested in the experimental data and showed its suitability to describe the mobilization of trapped nonwetting phase in fractures.

\section{Nomenclature}

$A=$ area fraction of a pixel, $\mathrm{m}^{2}$

$b=$ resolution (width of pixel), $\mathrm{m}$

$d=$ aperture, $10^{-6} \mathrm{~m}$

$d_{b}=$ pore-body aperture, $10^{-6} \mathrm{~m}$

$d_{H}=$ hydraulic aperture, $10^{-6} \mathrm{~m}$

$d_{t}=$ pore-throat aperture, $10^{-6} \mathrm{~m}$

$d_{Z}=$ gap distance between the highest point of the rough plate and the flat top plate, $10^{-6} \mathrm{~m}$ 
$\bar{d}=$ mean aperture, $10^{-6} \mathrm{~m}$

$g(d)=$ conductivity $\left(d^{3}\right)$ of aperture $d$ at a particular location, $\mathrm{m}^{3}$

$g_{m}=$ effective conductivity of the medium, $\mathrm{m}^{3}$

$k=$ permeability, $\mathrm{m}^{2}$

$k_{f}=$ fracture permeability, $\mathrm{m}^{2}$

$L_{\text {cor }}=$ correlation length of the aperture, $10^{-6} \mathrm{~m}$

$L_{g}=$ length of a ganglion, $10^{-6} \mathrm{~m}$

$L_{p}=$ length of the pore, $10^{-6} \mathrm{~m}$

$n(d)=$ area fraction from the histogram of a surface as a function of aperture, $\mathrm{m}^{2}$

$N_{c a}=$ capillary number, dimensionless

$N_{y}=$ number of pixels perpendicular to flow, dimensionless

$P_{c}=$ capillary pressure, $\mathrm{N} / \mathrm{m}^{2}$

$Q=$ volumetric-flow rate, $\mathrm{m}^{3} / \mathrm{s}$

$S_{a}=$ arithmetic deviation from average height, dimensionless

$S_{q}=$ root-mean-square deviation from average height, dimensionless

$v=$ superficial velocity, $\mathrm{m} / \mathrm{s}$

$w=$ width of the fracture perpendicular to flow, $\mathrm{m}$

$Z=$ coordination number, dimensionless

$\gamma=\mathrm{IFT}, \mathrm{N} / \mathrm{m}$

$\Delta P=$ capillary pressure difference, $\mathrm{N} / \mathrm{m}^{2}$

$\theta=$ contact angle, dimensionless

$\mu=$ displacing-fluid viscosity, Pa.s

$\mu_{w}=$ water viscosity, $\mathrm{Pa} \cdot \mathrm{s}$

$\nabla P=$ pressure gradient, $\mathrm{N} / \mathrm{m}^{2} / \mathrm{m}$

\section{Acknowledgments}

The authors acknowledge Saudi Aramco for providing the scholarship for author B. I. AlQuaimi for his PhD degree, and also acknowledge the generous support provided by the sponsors of the Joint Industry Project on Foam for Enhanced Oil Recovery at Delft University of Technology. Additional data are made available through AlQuaimi and Rossen (2017).

\section{References}

AlQuaimi, B. I. and Rossen, W. R. 2017. Capillary Desaturation Curve Additional Figures. Delft University of Technology Repository, Data Set, https:// doi.org/10.4121/uuid:b959da2e-f955-4991-b2bc-b2514397671f.

Brown, S. R. and Scholz, C. H. 1985. Broad Bandwidth Study of the Topography of Natural Rock Surfaces. J. Geophys. Res. 90 (B14): 12575-12582. https://doi.org/10.1029/JB090iB14p12575.

Brown, S. R., Kranz, R. L., and Bonner, B. P. 1986. Correlation Between the Surfaces of Natural Rock Joints. Geophys. Res. Lett. 13 (13): $1430-1433$. https://doi.org/10.1029/GL013i013p01430.

Buchgraber, M., Castanier, L. M., and Kovscek, A. R. 2012. Microvisual Investigation of Foam Flow in Ideal Fractures: Role of Fracture Aperture and Surface Roughness. Presented at the SPE Annual Technical Conference and Exhibition, San Antonio, Texas, 8-10 October. SPE-159430-MS. https:// doi.org/10.2118/159430-MS.

Chatzis, I. and Morrow, N. R. 1984. Correlation of Capillary Number Relationships for Sandstone. SPE J. 24 (5): 555-562. SPE-10114-PA. https:// doi.org/10.2118/10114-PA.

Chatzis, I., Morrow, N. R., and Lim, H. T. 1983. Magnitude and Detailed Structure of Residual Oil Saturation. SPE J. 23 (2): 311-326. SPE-10681-PA. https://doi.org/10.2118/10681-PA.

Chen, C.-Y., Horne, R. N., and Fourar, M. 2004a. Experimental Study of Liquid-Gas Flow Structure Effects on Relative Permeabilities in a Fracture. Water Resour. Res. 40 (8): W08301, 15 pages. https://doi.org/10.1029/2004WR003026.

Chen, C.-Y., Li, K., and Horne, R. N. 2004b. Experimental Study of Phase Transformation Effects on Relative Permeabilities in Fractures. Presented at the SPE Annual Technical Conference and Exhibition, Houston, 26-29 September. SPE-90233-MS. https://doi.org/10.2118/90233-MS.

Fourar, M., Bories, S., and Lenormand, R. 1992. Experimental Study of Two-Phase Flow in Rough Fractures. Proc., Seventeenth Workshop on Geothermal Reservoir Engineering, Stanford, California, 29-31 January, 215-218.

Gauteplass, J., Chaudhary, K., Kovscek, A. R. et al. 2015. Pore-Level Foam Generation and Flow for Mobility Control in Fractured Systems. Colloid. Surface. A. 468 (5 March): 184-192. https://doi.org/10.1016/j.colsurfa.2014.12.043.

Hakami, E., and Larsson, E. 1996. Aperture Measurements and Flow Experiments on a Single Natural Fracture. Int. J. Rock Mech. Min. 33 (4): $395-404$. https://doi.org/10.1016/0148-9062(95)00070-4.

Haugen, A., Fern $\varnothing$, M. A., Graue, A. et al. 2012. Experimental Study of Foam Flow in Fractured Oil-Wet Limestone for Enhanced Oil Recovery. SPE Res Eval \& Eng 15 (2): 218-228. SPE-129763-PA. https://doi.org/10.2118/129763-PA.

Hughes, R. G. and Blunt, M. J. 2001. Network Modeling of Multiphase Flow in Fractures. Adv. Water Resour. 24 (3-4): 409-421. https://doi.org/ 10.1016/S0309-1708(00)00064-6.

Izadi, M., Shadizadeh, S. R., and Moradi, S. 2012. Experimentally Measurements of Relative Permeability in Fractured Core. Int. J. Sci. Emerging Tech. 3 (1): 46-51.

Johns, R. A., Steude, J. S., Castanier, L. M. et al. 1993. Nondestructive Measurements of Fracture Aperture in Crystalline Rock Cores Using X-Ray Computed Tomography. J. Geophys. Res.-Sol.Ea. 98 (B2): 1889-1900. https://doi.org/10.1029/92JB02298.

Karpyn, Z., Grader, A., and Halleck, P. 2007. Visualization of Fluid Occupancy in a Rough Fracture Using Micro-Tomography. J. Colloid Interf. Sci. 307 (1): 181-187. https://doi.org/10.1016/j.jcis.2006.10.082.

Kirkpatrick, S. 1973. Percolation and Conduction. Rev. Modern Phys. 45 (4): 574-588.

Koplik, J. 1981. On the Effective Medium Theory of Random Networks. J. Phys. C. Solid State 14 (32): 4821. https://doi.org/10.1088/0022-3719/ 14/32/018.

Koplik, J. 1982. Creeping Flow in Two-Dimensional Networks. J. Fluid. Mech. 119 (June): 219-247. https://doi.org/10.1017/S0022112082001323.

Lake, L. W., Johns, R., Rossen, W. et al. 1986. Fundamentals of Enhanced Oil Recovery. Richardson, Texas: Society of Petroleum Engineers. 
Lang, P. S., Paluszny, A., and Zimmerman, R. 2015. Hydraulic Sealing due to Pressure Solution Contact Zone Growth in Siliciclastic Rock Fractures. J. Geophys. Res.-Sol. Ea. 120 (6): 4080-4101. https://doi.org/10.1002/2015JB011968.

Lang, P. S., Paluszny, A., and Zimmerman, R. W. 2016. Evolution of Fracture Normal Stiffness due to Pressure Dissolution and Precipitation. Int. J. Rock Mech. Min. 88 (October): 12-22. https://doi.org/10.1016/j.ijrmms.2016.06.004.

Larson, R. G., Davis, H. T., and Scriven, L. E. 1981. Displacement of Residual Nonwetting Fluid from Porous Media. Chem. Eng. Sci. 36 (1): $75-85$. https://doi.org/10.1016/0009-2509(81)80049-8.

Maloney, D. and Doggett, K. 1997. Multiphase Flow in Fractures. Report Number SCA-9730, US Department of Energy under contract DE-AC22-94PC91008.

Mayer, A. S. and Miller, C. T. 1992. The Influence of Porous Medium Characteristics and Measurement Scale on Pore-Scale Distributions of Residual Nonaqueous-Phase Liquids. J. Contam. Hydrol. 11 (3-4): 189-213. https://doi.org/10.1016/0169-7722(92)90017-9.

Moore, T. and Slobod, R. 1955. Displacement of Oil by Water-Effect of Wettability, Rate, and Viscosity on Recovery. Presented at the Fall Meeting of the Petroleum Branch of AIME, New Orleans, 2-5 October. SPE-502-G. https://doi.org/10.2118/502-G.

Morrow, N. R., Brower, K. R., Ma, S. et al. 1990. Fluid Flow in Healed Tectonic Fractures. J Pet Technol 42 (10): 1310-1318. SPE-19096-PA. https:// doi.org/10.2118/19096-PA.

Nelson, R. 2001. Geologic Analysis of Naturally Fractured Reservoirs. Houston: Gulf Professional Publishing.

Odling, N. E. and Roden, J. E. 1997. Contaminant Transport in Fractured Rocks with Significant Matrix Permeability, Using Natural Fracture Geometries. J. Contam. Hydrol. 27 (3-4): 263-283. https://doi.org/10.1016/S0169-7722(96)00096-4.

Olsson, R. and Barton, N. 2001. An Improved Model for Hydromechanical Coupling During Shearing of Rock Joints. Int. J. Rock Mech. Min. 38 (3): 317-329. https://doi.org/10.1016/S1365-1609(00)00079-4.

Oron, A. P. and Berkowitz, B. 1998. Flow in Rock Fractures: The Local Cubic Law Assumption Reexamined. Water Resour. Res. 34 (11): $2811-2825$. https://doi.org/10.1029/98WR02285.

Persoff, P. and Pruess, K. 1995. Two-Phase Flow Visualization and Relative Permeability Measurement in Natural Rough-Walled Rock Fractures. Water Resour. Res. 31 (5): 1175-1186. https://doi.org/10.1029/95WR00171.

Pieters, D. A. and Graves, R. M. 1994. Fracture Relative Permeability: Linear or Non-Linear Function of Saturation. Presented at the International Petroleum Conference and Exhibition of Mexico, Veracruz, Mexico, 10-13 October. SPE-28701-MS. https://doi.org/10.2118/28701-MS.

Pruess, K. and Tsang, Y. W. 1990. On Two-Phase Relative Permeability and Capillary Pressure of Rough-Walled Rock Fractures. Water Resour. Res. 26 (9): 1915-1926. https://doi.org/10.1029/WR026i009p01915.

Pyrak-Nolte, L. J., Cook, N. G., and Nolte, D. D. 1988. Fluid Percolation Through Single Fractures. Geophys. Res. Lett. 15 (11): $1247-1250$. https:// doi.org/10.1029/GL015i011p01247.

Qian, J., Chen, Z., Zhan, H. et al. 2011. Experimental Study of the Effect of Roughness and Reynolds Number on Fluid Flow in Rough-Walled Single Fractures: A Check of Local Cubic Law. Hydrol. Process 25 (4): 614-622. https://doi.org/10.1002/hyp.7849.

Rabbani, A., Jamshidi, S., and Salehi, S. 2014. An Automated Simple Algorithm for Realistic Pore Network Extraction from Micro-Tomography Images. J. Pet. Sci. Eng. 123 (November): 164-171. https://doi.org/10.1016/j.petrol.2014.08.020.

Ramspott, L., Ballou, L., Carlson, R. et al. 1979. Technical Concept for Test of Geologic Storage of Spent Reactor Fuel in the Climax Granite, Nevada Test Site. Report UCRL-52796, International Nuclear Information System and International Atomic Energy Agency, June 1979.

Reed, R. L. and Healy, R. N. 1977. Some Physicochemical Aspects of Microemulsion Flooding: A Review. Proc., 1976 AIChE Symposium on Improved Oil Recovery by Surfactant and Polymer Flooding, Kansas City, Kansas, 12-14 April, 383-437. https://doi.org/10.1016/B978-0-12-641750-0.50017-7.

Romm, E. 1966. Fluid Flow in Fractures. Moscow: Nedra Publishing House.

Rossen, W. and Kumar, A. T. 1992. Single- and Two-Phase Flow in Natural Fractures. Presented at the SPE Annual Technical Conference and Exhibition, Washington, DC, 4-7 October. SPE-24915-MS. https://doi.org/10.2118/24915-MS.

Schrauf, T. and Evans, D. 1986. Laboratory Studies of Gas Flow Through a Single Natural Fracture. Water Resour. Res. 22 (7): 1038-1050. https:// doi.org/10.1029/WR022i007p01038.

Sheng, J. 2010. Modern Chemical Enhanced Oil Recovery: Theory and Practice. Houston: Gulf Professional Publishing.

Skoreyko, F. A., Villavicencio, A. P., Rodriguez Prada, H. et al. 2012. Understanding Foam Flow With a New Foam EOR Model Developed From Laboratory and Field Data of the Naturally Fractured Cantarell Field. Presented at the SPE Improved Oil Recovery Symposium, Tulsa, 14-18 April. SPE153942-MS. https://doi.org/10.2118/153942-MS.

Taber, J. J. 1981. Research on Enhanced Oil Recovery: Past, Present and Future. In Surface Phenomena in Enhanced Oil Recovery, ed. D. O. Shah, Chap. 1, 13-52. New York: Springer.

Tsang, Y. 1984. The Effect of Tortuosity on Fluid Flow Through a Single Fracture. Water Resour. Res. 20 (9): 1209-1215. https://doi.org/10.1029/ WR020i009p01209.

Tsang, Y. 1992. Usage of "Equivalent Apertures" for Rock Fractures as Derived From Hydraulic and Tracer Tests. Water Resour. Res. 28 (5): 1451-1455. https://doi.org/10.1029/92WR00361.

van Golf-Racht, T. D. 1982. Fundamentals of Fractured Reservoir Engineering, Vol. 12, first edition. Amsterdam: Elsevier Science.

Wang, J. and Narasimhan, T. N. 1985. Hydrologic Mechanisms Governing Fluid Flow in a Partially Saturated, Fractured, Porous Medium. Water Resour. Res. 21 (12): 1861-1874. https://doi.org/10.1029/WR021i012p01861.

Witherspoon, P. A., Wang, J. S., Iwai, K. et al. 1980. Validity of Cubic Law for Fluid Flow in a Deformable Rock Fracture. Water Resour. Res. 16 (6): 1016-1024. https://doi.org/10.1029/WR016i006p01016.

Yan, W., Miller, C. A., and Hirasaki, G. J. 2006. Foam Sweep in Fractures for Enhanced Oil Recovery. Colloid. Surface. A 282-283 (20 July): $348-359$. https://doi.org/10.1016/j.colsurfa.2006.02.067.

Yeganeh, M., Hegner, J., Lewandowski, E. et al. 2016. Capillary Desaturation Curve Fundamentals. Presented at the SPE Improved Oil Recovery Conference, Tulsa, 11-13 April. SPE-179574-MS. https://doi.org/10.2118/179574-MS.

Zimmerman, R. W. and Bodvarsson, G. S. 1996. Hydraulic Conductivity of Rock Fractures. Transport Porous Med. 23 (1): 1-30. https://doi.org/ 10.1007/BF00145263. 


\section{Appendix A}

Topography of fracture samples with conceptual 2D network superimposed on images.

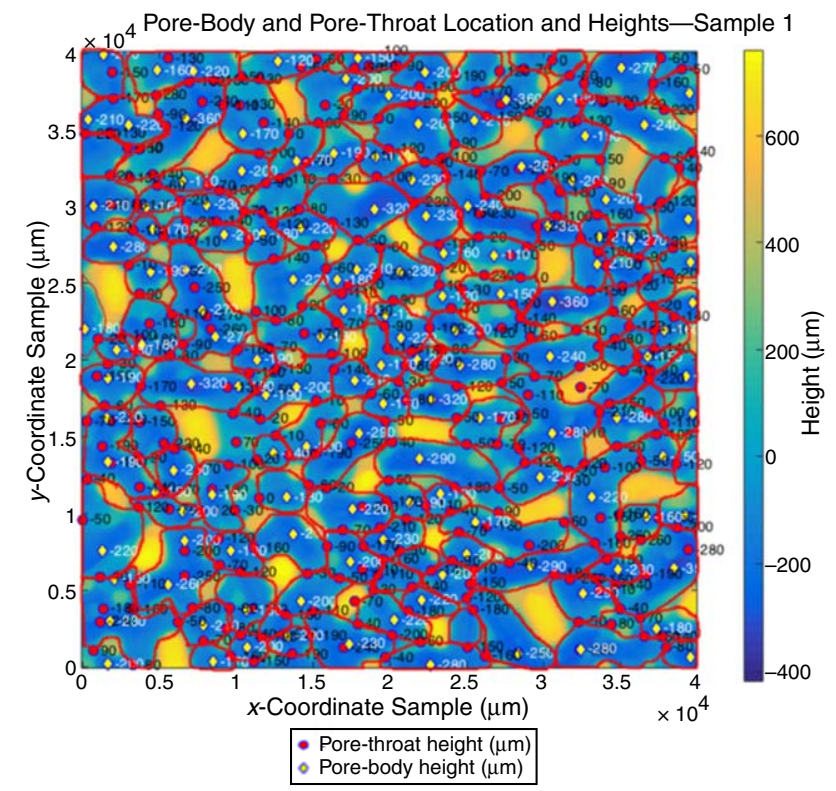

Pore-Body and Pore-Throat Location and Heights-Sample 2
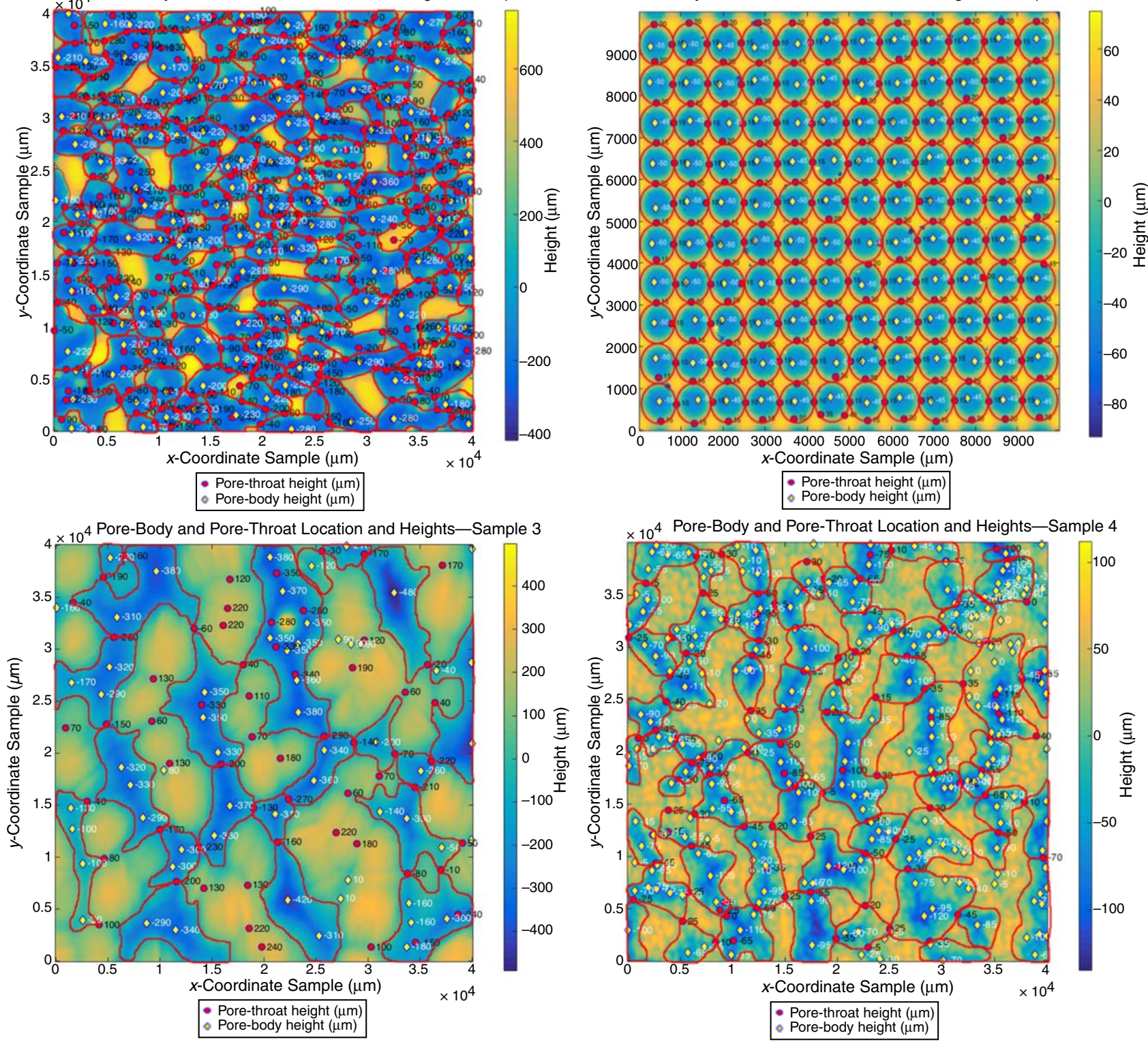

Pore-Body and Pore-Throat Location and Heights-Sample 5

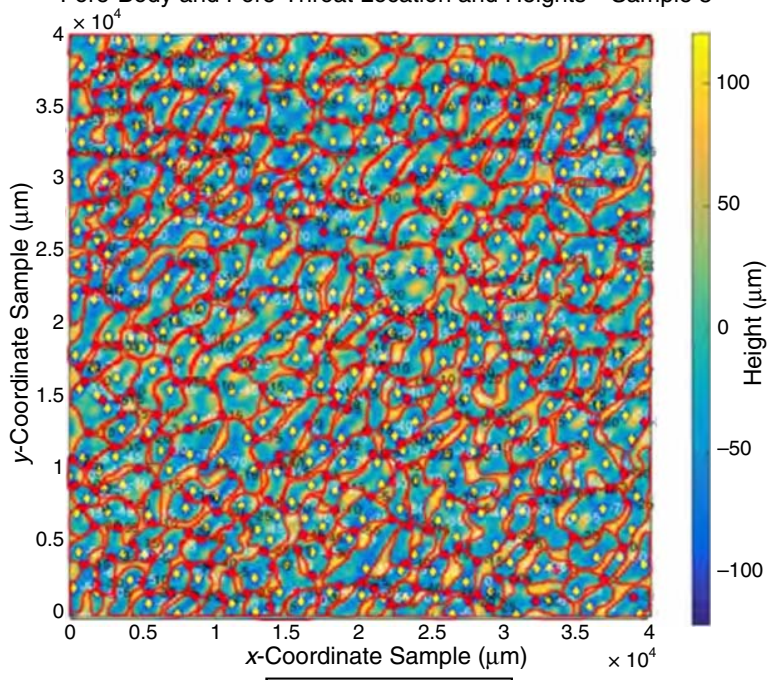

- Pore-throat height $(\mu \mathrm{m})$

- Pore-body height $(\mu \mathrm{m})$ 


\section{Appendix B}

Example of unprocessed images (Sample 4) and processed images (Sample 4).

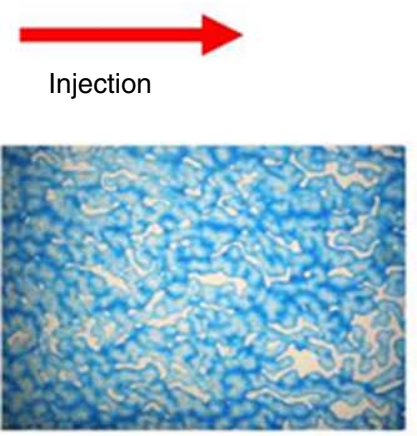

Unprocessed Images (Sample 4)
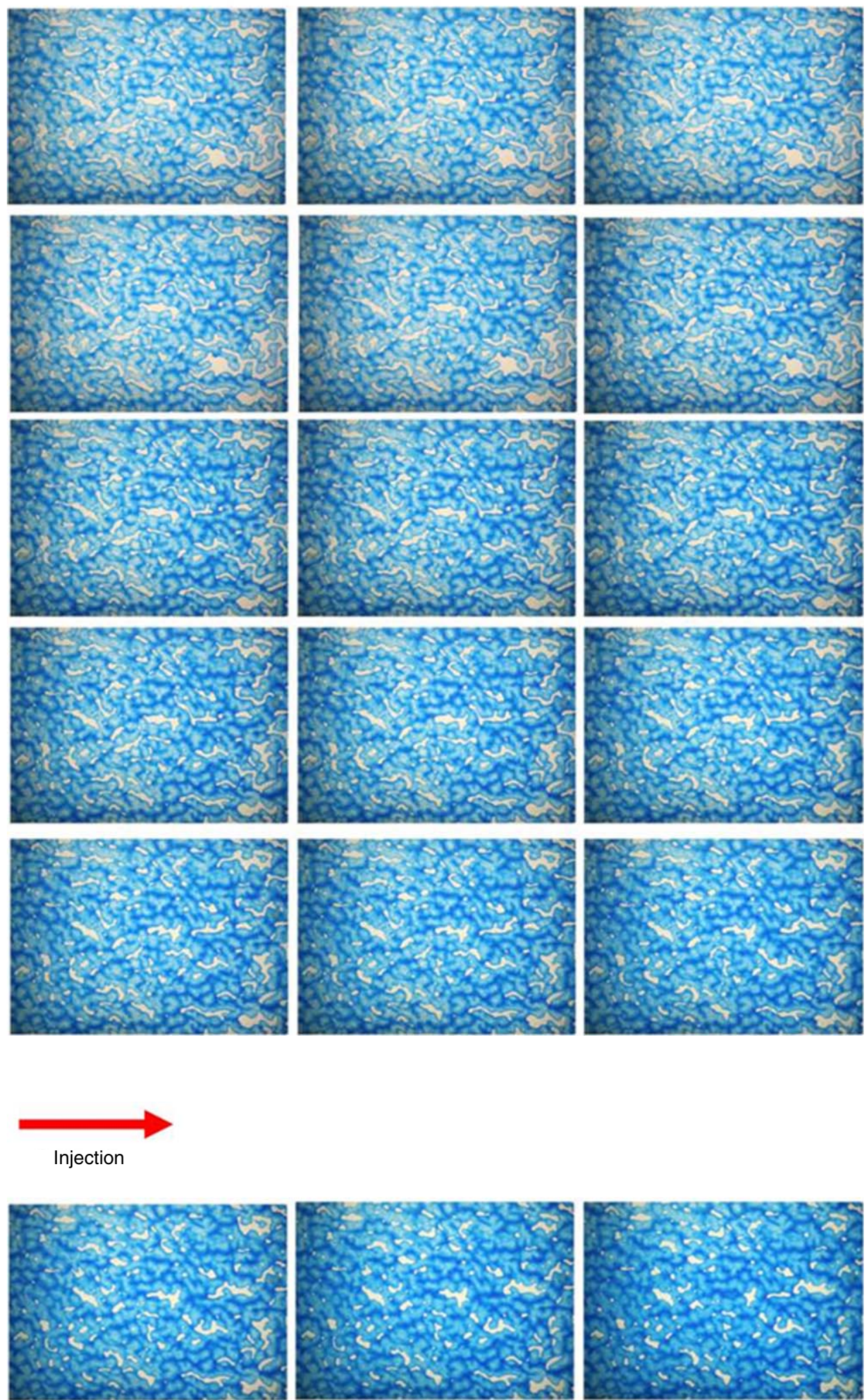


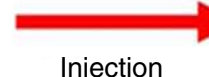

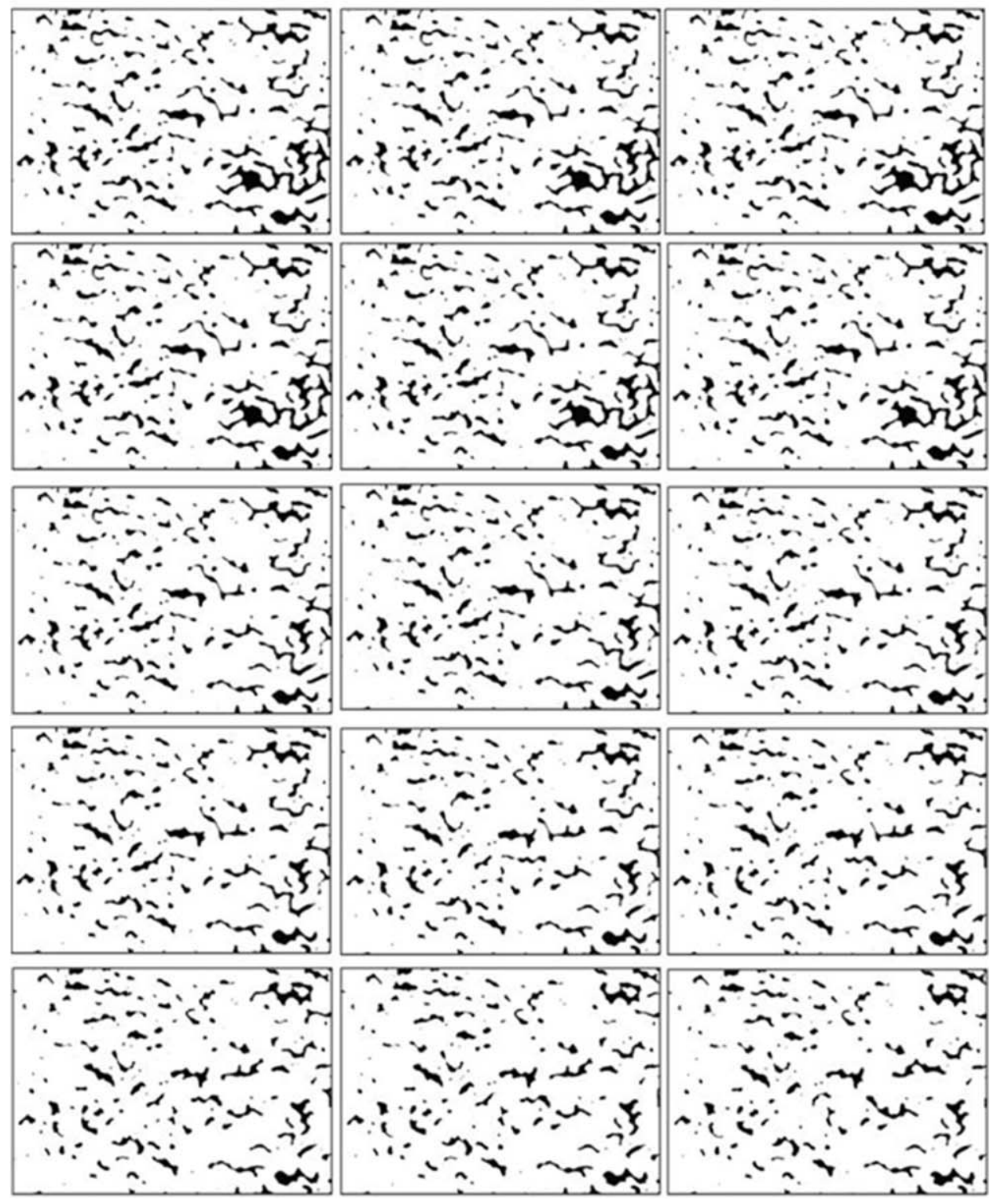

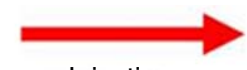

Injection

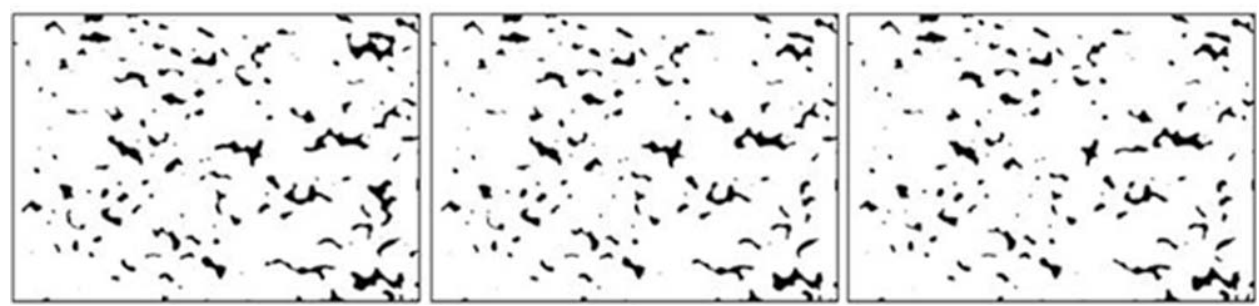

SI Metric Conversion Factors 
Bander I. AlQuaimi is a Saudi Aramco reservoir engineer, currently pursuing a PhD degree in petroleum engineering at Delft University of Technology, The Netherlands. He has worked in reservoir simulation, production engineering, reservoir characterization and modeling, and reservoir management. AlQuaimi's area of specialty is NFR development and management. His current area of research is foam flow in fractures for enhanced oil recovery. AlQuaimi has authored or coauthored several publications on naturally fractured reservoirs. He holds bachelor's and master's degrees in petroleum engineering from King Saud University, Saudi Arabia, and West Virginia University, respectively. AlQuaimi is a certified petroleum engineering professional by SPE.

William R. Rossen is a professor of reservoir engineering in the Department of Geoscience and Engineering at Delft University of Technology. His current research concerns are use of foams for diverting fluid flow in porous media, modeling complex transport processes in networks, and understanding flow in naturally fractured geological formations. Rossen has authored or coauthored more than 80 journal publications. He was named Best Instructor at Delft University of Technology in 2011 and was named an IOR Pioneer at the SPE/DOE Symposium on Improved Oil Recovery in 2012. Rossen is an SPE Distinguished Member. 